Revista de Matemática: Teoría y Aplicaciones 2003 10(1-2) : 173-186

CIMPA - UCR - CCSS ISSN: 1409-2433

\title{
AN ENUMERATIVE PROCEDURE FOR IDENTIFYING MAXIMAL COVERS
}

\author{
S. MUÑOZ*
}

Received: 21 Jan 2002

\begin{abstract}
Resumen
En este trabajo se presenta un procedimiento enumerativo que identifica todos los cubrimientos maximales respecto del conjunto de cubrimientos implicados por una restricción de tipo mochila con variables $0-1$. Las desigualdades inducidas por estos cubrimientos maximales no están dominadas por la desigualdad inducida por ningún otro cubrimiento implicado por la restricción de tipo mochila. Así pues, su identificación puede contribuir al reforzamiento de formulaciones de problemas de programación 0-1. Por otra parte, se presenta una mejora de un procedimiento de la literatura existente que únicamente identifica ciertos cubrimientos maximales. Además, se muestran algunos resultados computacionales comparativos en los que ambos procedimientos se han aplicado a restricciones de tipo mochila generadas aleatoriamente.
\end{abstract}

Palabras clave: Cubrimientos maximales, formulaciones más fuertes, restricciones de tipo mochila, desigualdades dominadas

\begin{abstract}
In this paper we present an enumerative procedure for identifying all maximal covers from the set of covers implied by a $0-1$ knapsack constraint. The inequalities induced by these maximal covers are not dominated by the inequality induced by any other cover implied by the knapsack constraint. Thus, their identification can help to tighten 0-1 models. On the other hand, we present an improvement on a procedure taken from the literature for identifying certain maximal covers. Some comparative computational experiments where both procedures have been applied to randomly generated knapsack constraints are also reported.
\end{abstract}

Keywords: Maximal covers, tighter formulations, knapsack constraints, dominated inequalities

Mathematics Subject Classification: 90C10,90C05

\footnotetext{
${ }^{*}$ Departamento de Estadística e Investigación Operativa I, Facultad de Ciencias Matemáticas, Universidad Complutense de Madrid, Ciudad Universitaria, 28040 Madrid, Spain. E-Mail: smunoz@estad.ucm.es
} 


\section{Introduction}

Consider the 0-1 linear programming problem

$$
\max \left\{\sum_{j \in J} c_{j} x_{j} \mid \sum_{j \in J} a_{i j} x_{j} \leq b_{i} \quad \forall i \in I, \quad x_{j} \in\{0,1\} \quad \forall j \in J\right\},
$$

where $J=\{1, \ldots, n\}, I=\{1, \ldots, m\}$ and $\left\{c_{j}\right\}_{j \in J},\left\{a_{i j}\right\}_{i \in I, j \in J},\left\{b_{i}\right\}_{i \in I}$ are real numbers.

The LP relaxation of $(P)$ is the same problem $(P)$ where each variable $x_{j}$ is allowed to take any value in the interval $[0,1]$.

We say that two constraint systems $\boldsymbol{A} \boldsymbol{x} \leq \boldsymbol{b}$ and $\boldsymbol{A}^{\prime} \boldsymbol{x} \leq \boldsymbol{b}^{\prime}$ are equivalent if $\{\boldsymbol{x} \in$ $\left.\{0,1\}^{n} \mid \boldsymbol{A} \boldsymbol{x} \leq \boldsymbol{b}\right\}=\left\{\boldsymbol{x} \in\{0,1\}^{n} \mid \boldsymbol{A}^{\prime} \boldsymbol{x} \leq \boldsymbol{b}^{\prime}\right\}$. The system $\boldsymbol{A}^{\prime} \boldsymbol{x} \leq \boldsymbol{b}^{\prime}$ is said to be as tight as the system $\boldsymbol{A} \boldsymbol{x} \leq \boldsymbol{b}$ if it is equivalent to $\boldsymbol{A} \boldsymbol{x} \leq \boldsymbol{b}$ and $\left\{\boldsymbol{x} \in[0,1]^{n} \mid \boldsymbol{A}^{\prime} \boldsymbol{x} \leq \boldsymbol{b}^{\prime}\right\} \subseteq$ $\left\{\boldsymbol{x} \in[0,1]^{n} \mid \boldsymbol{A} \boldsymbol{x} \leq \boldsymbol{b}\right\}$. The system $\boldsymbol{A}^{\prime} \boldsymbol{x} \leq \boldsymbol{b}^{\prime}$ is said to be tighter than the system $\boldsymbol{A} \boldsymbol{x} \leq \boldsymbol{b}$ if it is equivalent to $\boldsymbol{A} \boldsymbol{x} \leq \boldsymbol{b}$ and $\left\{\boldsymbol{x} \in[0,1]^{n} \mid \boldsymbol{A}^{\prime} \boldsymbol{x} \leq \boldsymbol{b}^{\prime}\right\} \subset\left\{\boldsymbol{x} \in[0,1]^{n} \mid \boldsymbol{A} \boldsymbol{x} \leq \boldsymbol{b}\right\}$.

An inequality $\sum_{j=1}^{n} a_{j} x_{j} \leq b$ is said to be valid for a set $R \subseteq \mathbb{R}^{n}$ if it is satisfied by any vector $\left(x_{1}, \ldots, x_{n}\right) \in R$.

The tighter a 0-1 model, the smaller could the gap be between the optimal values of the related 0-1 problem and its LP relaxation, and, probably, less computational effort could be required to solve the problem. Therefore, we are interested in finding tighter formulations for problem $(P)$. This can be done by using valid inequalities for its feasible region, e.g., inequalities induced by maximal covers from the set of covers implied by any constraint of $(P)$, see $[2,3,5,6,8,11]$ among others.

This paper is organized as follows: Section 2 reviews classical types of covers and states some results concerning them. Section 3 presents an enumerative procedure for identifying all maximal covers from the set of covers implied by a knapsack constraint. Section 4 proves that the non-dominated extensions considered in [1] are maximal covers, and it presents an improvement on the procedure given in [1] for identifying them. Section 5 reports some computational results for the procedures proposed in Sections 3 and 4. Finally, Section 6 draws some conclusions from this work.

\section{Covers. Basic concepts and results}

In this section we review some types of covers given in the literature, see $[1,4,8,9]$ among many others. We also state some results concerning these types of covers; their proofs can be found in $[4,8]$.

Given a set of variables $\left\{x_{1}, \ldots, x_{n}\right\}$ and a set $F \subseteq\{1, \ldots, n\}$, we define $X(F)=\sum_{j \in F} x_{j}$.

Definition 1. A cover $C$ is a set of indices of variables that induces the inequality $X\left(C^{+}\right)-X\left(C^{-}\right) \leq k_{C}-\left|C^{-}\right|$, where $C^{+} \cup C^{-}=C, C^{+} \cap C^{-}=\emptyset$ and $k_{C}$ is an integer such that $1 \leq k_{C} \leq|C|$.

Definition 2. A trivial cover is a cover $C$ such that $k_{C}=|C|$. 
Definition 3. A cover $C$ is said to be implied by the constraint $\sum_{j=1}^{n} a_{j} x_{j} \leq b$ if its induced inequality is valid for the set $\left\{\left(x_{1}, \ldots, x_{n}\right) \in\{0,1\}^{n} \mid \sum_{j=1}^{n} a_{j} x_{j} \leq b\right\}$.

We consider knapsack constraints from problem $(P)$ of the form

$$
\sum_{j \in J_{0}} a_{j} x_{j} \leq b
$$

where $0<a_{j} \leq b \quad \forall j \in J_{0}, \sum_{j \in J_{0}} a_{j}>b$ and $a_{j} \leq a_{j^{\prime}} \quad \forall j, j^{\prime} \in J_{0}$ such that $j<j^{\prime}$. (Note that any non-redundant constraint of $(P)$ can be put in this form.)

Given a non-empty set $C \subseteq J_{0}$, let $m_{l}(C)$ denote the set of the $l$ smallest indices of $C$, where $l$ is an integer such that $1 \leq l \leq|C|$.

Proposition 1. Let $C \subseteq J_{0}$ be a non-trivial cover with induced inequality $X(C) \leq k_{C}$. Then $C$ is implied by constraint (1) if and only if $\sum_{j \in m_{k_{C}+1}(C)} a_{j}>b$.

Definition 4. The inequality $\sum_{j=1}^{n} a_{j} x_{j} \leq b$ is said to be dominated by the inequality $\sum_{j=1}^{n} a_{j}^{\prime} x_{j} \leq b^{\prime}$ if $\left\{\left(x_{1}, \ldots, x_{n}\right) \in[0,1]^{n} \mid \sum_{j=1}^{n} a_{j}^{\prime} x_{j} \leq b^{\prime}\right\} \subseteq\left\{\left(x_{1}, \ldots, x_{n}\right) \in[0,1]^{n} \mid \sum_{j=1}^{n} a_{j} x_{j} \leq\right.$ $b\}$.

Definition 5. Given a set of covers $\mathcal{C}, C \in \mathcal{C}$ is a maximal cover from $\mathcal{C}$ if its induced inequality is not dominated by the inequality induced by $C^{\prime} \quad \forall C^{\prime} \in \mathcal{C}$ such that $C^{\prime+} \neq C^{+}$ or $C^{\prime-} \neq C^{-}$or $k_{C^{\prime}} \neq k_{C}$.

Proposition 2. Let $C$ be a maximal cover from the set of covers implied by constraint (1). Then $C$ is a non-trivial cover, $C \subseteq J_{0}$ and its induced inequality is $X(C) \leq \max \{l \mid$ $\left.\sum_{j \in m_{l}(C)} a_{j} \leq b\right\}$.

Definition 6. A non-trivial cover $C$ implied by constraint (1) with induced inequality $X(C) \leq k_{C}$ is said to be minimal with respect to constraint (1) if $\sum_{j \in C \backslash\{k\}} a_{j} \leq b \quad \forall k \in C$.

Proposition 3. If $C$ is a minimal cover with respect to constraint (1), then $C \subseteq J_{0}$ and $k_{C}=|C|-1$.

Given a non-empty set $C \subseteq J_{0}$, we define $\underline{\gamma}(C)=\min \{j \mid j \in C\}$ and $\bar{\gamma}(C)=\max \{j \mid$ $j \in C\}$. 
Proposition 4. A set $C \subseteq J_{0}$ is a minimal cover with respect to constraint (1) with induced inequality $X(C) \leq|C|-1$ if and only if $\sum_{j \in C} a_{j}>b$ and $\sum_{j \in C \backslash\{\underline{\gamma}(C)\}} a_{j} \leq b$.

Definition 7. Let $C$ be a minimal cover with respect to constraint (1). The extension of $C$ is the set $E(C)=C \cup\left\{j \in J_{0} \mid j>\bar{\gamma}(C)\right\}$.

Proposition 5. If $C$ is a minimal cover with respect to constraint (1), then

(1) $E(C)$ is a non-trivial cover implied by constraint (1), and the inequality $X(E(C)) \leq$ $|C|-1$ is induced by $E(C)$.

(2) The inequality induced by $C$ is dominated by the inequality $X(E(C)) \leq|C|-1$.

Theorem 1. If $C$ is a maximal cover from the set of covers implied by constraint (1), then there exists a unique minimal cover with respect to constraint (1), say $C^{\prime}$, such that $E\left(C^{\prime}\right)=C$.

Theorem 2. Let $C$ be a minimal cover with respect to constraint (1) and let $X(E(C)) \leq$ $|C|-1$ be the inequality induced by $E(C)$. Then $E(C)$ is a maximal cover from the set of covers implied by constraint (1) if and only if one of the two following conditions is satisfied:

(1) $E(C) \subset J_{0}$ and $\sum_{j \in C \backslash\{\bar{\gamma}(C)\}} a_{j}+a_{\bar{\gamma}\left(J_{0} \backslash E(C)\right)} \leq b$.

(2) $E(C)=J_{0}$.

\section{Identification of all maximal covers from the set of covers implied by a knapsack constraint}

For simplicity, from now on it will be assumed that $J_{0}=\left\{1, \ldots, n_{0}\right\}$.

$$
\begin{aligned}
& \text { Let } \underline{k}=\max \left\{l \in\left\{1, \ldots, n_{0}-1\right\} \mid \sum_{j=n_{0}-(l-1)}^{n_{0}} a_{j} \leq b\right\} \text { and } \bar{k}=\max \left\{l \in\left\{1, \ldots, n_{0}-1\right\} \mid\right. \\
& \left.\sum_{j=2}^{l+1} a_{j} \leq b\right\} \text {. }
\end{aligned}
$$

Lemma 1 states that $\underline{k}$ and $\bar{k}$ are, respectively, a lower and an upper bound for the righthand-side of the inequality induced by any minimal cover with respect to constraint (1). It can be shown that the lower bound $\underline{k}$ is always attainable and, under some assumptions, the upper bound $\bar{k}$ is attainable as well, see [8].

Lemma 1. If $C$ is a minimal cover with respect to constraint (1), then $\underline{k} \leq k_{C} \leq \bar{k}$ and, equivalently, $\underline{k}+1 \leq|C| \leq \bar{k}+1$. 
Proof. By Propositions 3 and 4 we have that $k_{C}=|C|-1, \sum_{j \in C} a_{j}>b$ and $\sum_{j \in C \backslash\{\underline{\gamma}(C)\}} a_{j} \leq$ $b$.

Suppose that $k_{C}<\underline{k}$. In this case $|C| \leq \underline{k}$, hence $\sum_{j \in C} a_{j} \leq \sum_{j=n_{0}-(\underline{k}-1)}^{n_{0}} a_{j} \leq b$, which is a contradiction.

Now, suppose that $k_{C}>\bar{k}$. Then $|C \backslash\{\underline{\gamma}(C)\}| \geq \bar{k}+1$ and, since $C \backslash\{\underline{\gamma}(C)\} \subseteq$ $\left\{2, \ldots, n_{0}\right\}$, it follows that $\sum_{j \in C \backslash\{\underline{\gamma}(C)\}} a_{j} \geq \sum_{j=2}^{\bar{k}+2} a_{j}>b$, which is also a contradiction.

Consequently, we must have $\underline{k} \leq k_{C} \leq \bar{k}$ and $\underline{k}+1 \leq|C| \leq \bar{k}+1$.

Given a cover $C=\left\{j_{1}, \ldots, j_{|C|}\right\}$, from now on it will be assumed that $j_{1}<\ldots<j_{|C|}$.

Definition 8. Let $C=\left\{j_{1}, \ldots, j_{|C|}\right\} \subseteq J_{0}$ and $C^{\prime}=\left\{j_{1}^{\prime}, \ldots, j_{|C|}^{\prime}\right\} \subseteq J_{0}$ be two distinct covers with the same cardinality such that $\sum_{j \in C} a_{j}>b$ and $\sum_{j \in C^{\prime}} a_{j}>b$, and let $k_{0}=\min \{k \in$ $\left.\{1, \ldots,|C|\} \mid j_{k} \neq j_{k}^{\prime}\right\}$. If $j_{k_{0}}<j_{k_{0}}^{\prime}, C$ is said to be previous to $C^{\prime}$ and $C^{\prime}$ is said to be subsequent to $C$.

Definition 9. Let $C$ and $C^{\prime}$ be two covers such that $C$ is previous to $C^{\prime}$. If there is not any cover subsequent to $C$ and previous to $C^{\prime}, C$ is said to be immediately previous to $C^{\prime}$ and $C^{\prime}$ is said to be immediately subsequent to $C$.

Given $k_{C} \in\{\underline{k}, \ldots, \bar{k}\}$, let $A_{k_{C}}=\left\{j_{1}, \ldots, j_{k_{C}+1}\right\}$, where $j_{k}=\min \left\{j \in J_{0} \mid j>\right.$ $\left.j_{k-1}, \sum_{l=1}^{k-1} a_{j_{l}}+a_{j}+\sum_{l=n_{0}-\left(k_{C}-k\right)}^{n_{0}} a_{l}>b\right\} \quad \forall k \in\left\{1, \ldots, k_{C}+1\right\}$ and $j_{0}=0$.

Lemma 2. Let $k_{C} \in\{\underline{k}, \ldots, \bar{k}\}$. Then every cover $C \subset J_{0}$ such that $|C|=k_{C}+1, C \neq$ $A_{k_{C}}$ and $\sum_{j \in C} a_{j}>b$, is subsequent to $A_{k_{C}}$.

Proof. It follows from the definition of $A_{k_{C}}$.

Definition 10. A consecutive cover is a cover $C=\left\{j_{1}, \ldots, j_{|C|}\right\}$ such that $j_{k+1}=j_{k}+1$ $\forall k \in\{1, \ldots,|C|-1\}$.

Proposition 6. Let $C \subset J_{0}$ be a consecutive cover such that $\sum_{j \in C} a_{j}>b$ and let $C^{\prime}$ be a minimal cover with respect to constraint (1) subsequent to $C$. Then $E\left(C^{\prime}\right)$ is not a maximal cover from the set of covers implied by constraint (1).

Proof. Let $C=\left\{j_{1}, \ldots, j_{|C|}\right\}$ and $C^{\prime}=\left\{j_{1}^{\prime}, \ldots, j_{|C|}^{\prime}\right\}$. Since $C$ is a consecutive cover and $C^{\prime}$ is a minimal cover with respect to constraint (1) subsequent to $C$, we have that 
$b \geq \sum_{j \in C^{\prime} \backslash\left\{\underline{\gamma}\left(C^{\prime}\right)\right\}} a_{j} \geq \sum_{j \in C \backslash\{\underline{\gamma}(C)\}} a_{j}$. So, by Proposition 4,C is a minimal cover with respect to constraint (1) and, by claim (1) of Proposition $5, E(C)$ is a cover implied by (1), and the inequality $X(E(C)) \leq|C|-1$ is induced by it. Now, by Proposition 2, the inequality induced by $E\left(C^{\prime}\right)$ is $X\left(E\left(C^{\prime}\right)\right) \leq|C|-1$ and, since $E\left(C^{\prime}\right) \subset E(C)$, the inequality $X\left(E\left(C^{\prime}\right)\right) \leq|C|-1$ is dominated by $X(E(C)) \leq|C|-1$. Thus, $E\left(C^{\prime}\right)$ is not a maximal cover from the set of covers implied by constraint (1).

Algorithm 1 identifies all maximal covers from the set of covers implied by constraint (1) by using an enumerative procedure based on Theorems 1 and 2, Lemmas 1 and 2, and Proposition 6.

\section{Algorithm 1.}

Step 1. Set $h=0, j_{0}=0, k_{C}=\max \left\{l \in\left\{1, \ldots, n_{0}-1\right\} \mid \sum_{j=n_{0}-(l-1)}^{n_{0}} a_{j} \leq b\right\}$ and $\bar{k}=\max \left\{l \in\left\{k_{C}, \ldots, n_{0}-1\right\} \mid \sum_{j=2}^{l+1} a_{j} \leq b\right\}$.

Step 2. Set $j_{k}=\min \left\{j \in J_{0} \mid j>j_{k-1}, \sum_{l=1}^{k-1} a_{j_{l}}+a_{j}+\sum_{l=n_{0}-\left(k_{C}-k\right)}^{n_{0}} a_{l}>b\right\} \quad \forall k \in$ $\left\{1, \ldots, k_{C}+1\right\}$ and $C=\left\{j_{1}, \ldots, j_{k_{C}+1}\right\}$.

Step 3. If the cover $C$ is not minimal with respect to constraint (1), go to Step 6 .

Step 4. If $E(C) \subset J_{0}$ and $\sum_{j \in C \backslash\{\bar{\gamma}(C)\}} a_{j}+a_{\bar{\gamma}\left(J_{0} \backslash E(C)\right)}>b$, go to Step 6 .

Step 5. Set $h=h+1$ and $C_{h}=E(C)$.

Step 6. If $C$ is a consecutive cover, go to Step 8.

Step 7. Let $C^{\prime}$ be the cover immediately subsequent to $C$. Set $C=C^{\prime}$ and go to Step 3 .

Step 8. If $k_{C}<\bar{k}$, set $k_{C}=k_{C}+1$ and go to Step 2. Otherwise, STOP (all maximal covers from the set of covers implied by constraint (1) have been identified).

EXAMPLE 1. Consider the 0-1 knapsack constraint

$$
2 x_{1}+3 x_{2}+4 x_{3}+6 x_{4}+8 x_{5} \leq 12
$$

Algorithm 1 proceeds as follows:

Step 1. $h=0, j_{0}=0, k_{C}=1, \bar{k}=2$

Step 2. $j_{1}=4, j_{2}=5, C=\{4,5\}$

Step 5. $h=1, C_{1}=\{4,5\}$

Step 8. $k_{C}=2$ 
Step 2. $j_{1}=1, j_{2}=2, j_{3}=5, C=\{1,2,5\}$

Step 5. $h=2, C_{2}=\{1,2,5\}$

Step 7. $C=\{1,3,5\}$

Step 5. $h=3, C_{3}=\{1,3,5\}$

Step 7. $C=\{1,4,5\}$

Step 7. $C=\{2,3,4\}$

Step 5. $h=4, C_{4}=\{2,3,4,5\}$

Accordingly, the maximal covers from the set of covers implied by constraint (2) are $C_{1}=\{4,5\}, C_{2}=\{1,2,5\}, C_{3}=\{1,3,5\}$ and $C_{4}=\{2,3,4,5\}$, and their induced inequalities are:

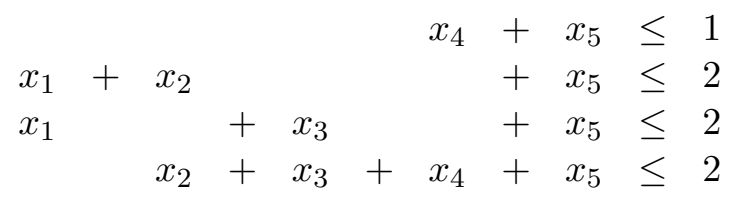

\section{Identification of maximal covers by using consecutive minimal covers and alternates}

In this section we review the concept of alternate of a minimal cover as well as some results given in [1], see also [7]. We prove that the non-dominated extensions considered in [1] are maximal covers from the set of covers implied by a knapsack constraint. We also present an improvement on the procedure proposed in [1] for identifying this type of maximal covers. This improvement consists in defining a set $m(C)$ that allows us to obtain more maximal covers.

Definition 11. Let $C$ be a minimal cover with respect to constraint (1). The alternate of $C$ is the set $\alpha(C)=\left\{l \in J_{0} \mid l<\underline{\gamma}(C), a_{l}+\sum_{j \in C \backslash\{\underline{\gamma}(C)\}} a_{j}>b\right\}$.

Given a minimal cover with respect to constraint (1), say $C$, we define $m(C)=\{k \in C \mid$ $\left.a_{k}=a_{\underline{\gamma}(C)}\right\}$.

Lemma 3. Let $C$ be a minimal cover with respect to constraint (1), let $l \in \alpha(C)$ and let $k \in m(C)$. Then

(1) $(C \backslash\{k\}) \cup\{l\}$ is a minimal cover with respect to constraint (1), and its induced inequality is $X((C \backslash\{k\}) \cup\{l\}) \leq|C|-1$.

(2) $E((C \backslash\{k\}) \cup\{l\})$ is a non-trivial cover implied by constraint (1), and the inequality $X(E((C \backslash\{k\}) \cup\{l\})) \leq|C|-1$ is induced by $E((C \backslash\{k\}) \cup\{l\})$.

(3) $(E(C) \backslash\{k\}) \cup\{l\}=E((C \backslash\{k\}) \cup\{l\})$ if and only if $k<\bar{\gamma}(C)$. 
Proof. (1) It follows from Propositions 3 and 4.

(2) It follows from claim (1) above and from claim (1) of Proposition 5.

(3) If $k<\bar{\gamma}(C)$, it is obvious that $(E(C) \backslash\{k\}) \cup\{l\}=E((C \backslash\{k\}) \cup\{l\})$. If $k=\bar{\gamma}(C)$, then $(E(C) \backslash\{k\}) \cup\{l\} \subset E(C) \cup\{l\} \subseteq E((C \backslash\{k\}) \cup\{l\})$.

Given a consecutive minimal cover $C$ with respect to constraint (1) such that $\underline{\gamma}(C)>1$, we define $\underline{C}=\{\underline{\gamma}(C)-1, \ldots, \bar{\gamma}(C)-1\}$.

Theorem 3. Let $C$ be a consecutive minimal cover with respect to constraint (1) and let $X(E(C)) \leq|C|-1$ be the inequality induced by $E(C)$. Then $E(C)$ is a maximal cover from the set of covers implied by constraint (1) if and only if one of the two following conditions is satisfied:

(1) $\underline{\gamma}(C)>1$ and $\sum_{j \in \underline{C}} a_{j} \leq b$.

(2) $\underline{\gamma}(C)=1$.

Proof. It follows from Theorem 2.

Lemma 4. Let $C$ be a consecutive minimal cover with respect to constraint (1) such that $\underline{\gamma}(C)>1$. If $\sum_{j \in \underline{C}} a_{j} \leq b$ and $\bar{\gamma}(C) \in m(C)$, then $\alpha(C)=\emptyset$.

Proof. It is obvious, since $a_{\underline{\gamma}(C)-1}+\sum_{j \in C \backslash\{\underline{\gamma}(C)\}} a_{j}=\sum_{j \in \underline{C}} a_{j} \leq b$.

Lemma 5. Let $C$ be a consecutive minimal cover with respect to constraint (1) such that $\underline{\gamma}(C)>1$. If $\sum_{j \in \underline{C}} a_{j}>b$, then

(1) $\underline{C}$ is a consecutive minimal cover with respect to constraint (1), and its induced inequality is $X(\underline{C}) \leq|C|-1$.

(2) $\alpha(\underline{C}) \cup\{\underline{\gamma}(\underline{C})\} \subseteq \alpha(C)$.

(3) If $\bar{\gamma}(C) \in m(C)$, then $\alpha(C)=\alpha(\underline{C}) \cup\{\underline{\gamma}(\underline{C})\}$.

Proof. (1) It follows from Propositions 3 and 4.

(2) Let $l \in \alpha(\underline{C}) \cup\{\underline{\gamma}(\underline{C})\}$. If $l \in \alpha(\underline{C})$, then $a_{l}+\sum_{j \in C \backslash\{\underline{\gamma}(C)\}} a_{j} \geq a_{l}+\sum_{j \in \underline{C} \backslash\{\underline{\gamma}(\underline{C})\}} a_{j}>b$. If $l=\underline{\gamma}(\underline{C})$, then $a_{l}+\sum_{j \in C \backslash\{\underline{\gamma}(C)\}} a_{j} \geq \sum_{j \in \underline{C}} a_{j}>b$. Therefore $l \in \alpha(C)$.

(3) By claim (2) above, it suffices to prove that $\alpha(C) \subseteq \alpha(\underline{C}) \cup\{\underline{\gamma}(\underline{C})\}$.

Let $l \in \alpha(C)$. Then $l \leq \underline{\gamma}(\underline{C})$. Now, if $l<\underline{\gamma}(\underline{C})$, we have that $a_{l}+\sum_{j \in \underline{C} \backslash\{\underline{\gamma}(\underline{C})\}} a_{j}=$ $a_{l}+\sum_{j \in C \backslash\{\underline{\gamma}(C)\}} a_{j}>b$, hence $l \in \alpha(\underline{C})$. 
Proposition 7. Let $C$ be a consecutive minimal cover with respect to constraint (1), let $l \in \alpha(C)$, let $k \in m(C)$ and let $X((E(C) \backslash\{k\}) \cup\{l\}) \leq|C|-1$ be the inequality induced by $(E(C) \backslash\{k\}) \cup\{l\}$. If $\sum_{j \in \underline{C}} a_{j}>b$ and $l \in \alpha(\underline{C}) \cup\{\underline{\gamma}(\underline{C})\}$, then $(E(C) \backslash\{k\}) \cup\{l\}$ is a non-trivial cover implied by constraint (1), but it is not a maximal cover from the set of covers implied by constraint (1).

Proof. If $k<\bar{\gamma}(C)$, by claims (2) and (3) of Lemma 3 it follows that $(E(C) \backslash\{k\}) \cup\{l\}=$ $E((C \backslash\{k\}) \cup\{l\})$ and it is a non-trivial cover implied by constraint (1). Now, since $E((C \backslash$ $\{k\}) \cup\{l\}) \subset J_{0}$ and $\sum_{j \in(C \backslash\{k, \bar{\gamma}(C)\}) \cup\{l\}} a_{j}+a_{k}=a_{l}+\sum_{j \in C \backslash\{\bar{\gamma}(C)\}} a_{j}=a_{l}+\sum_{j \in \underline{C} \backslash\{\underline{\gamma}(\underline{C})\}} a_{j}>b$, by Theorem 2 we have that $(E(C) \backslash\{k\}) \cup\{l\}$ is not a maximal cover from the set of covers implied by constraint (1).

If $k=\bar{\gamma}(C)$, considering that $\sum_{j \in m_{|C|}((E(C) \backslash\{k\}) \cup\{l\})} a_{j}=a_{l}+\sum_{j \in C \backslash\{\bar{\gamma}(C)\}} a_{j}=a_{l}+$ $\sum_{j \in C \backslash\{\underline{\gamma}(C)\}} a_{j}>b$, from Proposition 1 we can conclude that $(E(C) \backslash\{k\}) \cup\{l\}$ is a non-trivial cover implied by constraint (1). On the other hand, since $E((C \backslash\{k\}) \cup\{l\})=E(C) \cup\{l\}$, by claim (2) of Lemma 3 it follows that $E(C) \cup\{l\}$ is a non-trivial cover implied by constraint (1), and the inequality $X(E(C) \cup\{l\}) \leq|C|-1$ is induced by it. Now, it is obvious that $(E(C) \backslash\{k\}) \cup\{l\} \subset E(C) \cup\{l\}$ and, consequently, the inequality $X((E(C) \backslash\{k\}) \cup\{l\}) \leq|C|-1$ is dominated by $X(E(C) \cup\{l\}) \leq|C|-1$. So, $(E(C) \backslash\{k\}) \cup\{l\}$ is not a maximal cover from the set of covers implied by constraint (1).

Theorem 4. Let $C$ be a consecutive minimal cover with respect to constraint (1), let $l \in \alpha(C)$, let $k \in m(C)$ and let $X((E(C) \backslash\{k\}) \cup\{l\}) \leq|C|-1$ be the inequality induced by $(E(C) \backslash\{k\}) \cup\{l\}$. Then $(E(C) \backslash\{k\}) \cup\{l\}$ is a maximal cover from the set of covers implied by constraint (1) if and only if one of the two following conditions is satisfied:

(1) $\sum_{j \in \underline{C}} a_{j} \leq b$.

(2) $\sum_{j \in \underline{C}} a_{j}>b$ and $l \notin \alpha(\underline{C}) \cup\{\underline{\gamma}(\underline{C})\}$.

ProOF. $(\Rightarrow)$ It follows from Proposition 7 .

$(\Leftarrow)$ If condition $(1)$ is satisfied, by Lemma 4 we have that $\bar{\gamma}(C) \notin m(C)$ and, on the other hand, $a_{l}+\sum_{j \in \underline{C} \backslash\{\underline{\gamma}(\underline{C})\}} a_{j} \leq \sum_{j \in \underline{C}} a_{j} \leq b$. If condition (2) is satisfied, from claim (3) of Lemma 5 we can conclude that $\bar{\gamma}(C) \notin m(C)$ and, considering that $l<\underline{\gamma}(\underline{C})$ and $l \notin \alpha(\underline{C})$, it follows that $a_{l}+\sum_{j \in \underline{C} \backslash\{\underline{\gamma}(\underline{C})\}} a_{j} \leq b$. Thus, $k<\bar{\gamma}(C)$ and $a_{l}+\sum_{j \in \underline{C} \backslash\{\underline{\gamma}(\underline{C})\}} a_{j} \leq$ $b$ in both cases. Accordingly, by claim (3) of Lemma 3 and Theorem 2 we have that 
$(E(C) \backslash\{k\}) \cup\{l\}$ is a maximal cover from the set of covers implied by constraint (1), since $E((C \backslash\{k\}) \cup\{l\}) \subset J_{0}$ and $\sum_{j \in(C \backslash\{k, \bar{\gamma}(C)\}) \cup\{l\}} a_{j}+a_{k}=a_{l}+\sum_{j \in \underline{C} \backslash\{\underline{\gamma}(\underline{C})\}} a_{j}$.

Given a consecutive minimal cover with respect to constraint (1), say $C$, let $E_{M}(C)$ denote the set of maximal covers that are obtained by applying Theorems 3 and 4 to $C$, and let

$\underline{\alpha}(C)=\min \{l \mid l \in \alpha(C) \cup\{\underline{\gamma}(C)\}\}$. If $\underline{\gamma}(C)>1$, let $\bar{\alpha}(C)=\left\{\begin{array}{ll}\underline{\gamma}(C)-1 \text { if } \sum_{j \in \underline{C}} a_{j} \leq b \\ \underline{\alpha}(\underline{C})-1 \text { if } \sum_{j \in \underline{C}} a_{j}>b\end{array}\right.$.

Note. The inequality induced by any cover $F \in E_{M}(C)$ is $X(F) \leq \bar{\gamma}(C)-\underline{\gamma}(C)$.

Proposition 8. If $C$ is a consecutive minimal cover with respect to constraint (1), then

$E_{M}(C)= \begin{cases}\{E(C)\} & \text { if } \underline{\gamma}(C)=1 \\ \{E(C)\} \cup\{(E(C) \backslash\{k\}) \cup\{l\}\}_{l \in\{\underline{\alpha}(C), \ldots, \bar{\alpha}(C)\}, k \in m(C)} & \text { if } \underline{\gamma}(C)>1 \text { and } \bar{\alpha}(C)=\underline{\gamma}(C)-1 \\ \{(E(C) \backslash\{k\}) \cup\{l\}\}_{l \in\{\underline{\alpha}(C), \ldots, \bar{\alpha}(C)\}, k \in m(C)} & \text { if } \underline{\gamma}(C)>1 \text { and } \bar{\alpha}(C)<\underline{\gamma}(C)-1\end{cases}$

Proof. It follows from the definition of $\underline{\alpha}(C)$ and $\bar{\alpha}(C)$.

Lemma 6. Let $C$ be a consecutive minimal cover with respect to constraint (1) and let $F \in E_{M}(C)$. Then $F$ is a consecutive cover if and only if $F=E(C)$.

Proof. If $F \neq E(C)$, then by Proposition 8 we have that $F=(E(C) \backslash\{k\}) \cup\{l\}$, where $l \in\{\underline{\alpha}(C), \ldots, \bar{\alpha}(C)\}$ and $k \in m(C)$.

Suppose that $\bar{\gamma}(C) \in m(C)$. In this case, by Lemma 4 and claim (3) of Lemma 5 it follows that $\bar{\alpha}(C)<\underline{\alpha}(C)$, which is a contradiction. Therefore we must have $k<\bar{\gamma}(C)$ and, consequently, $F$ is a non-consecutive cover, since $l<k, l, \bar{\gamma}(C) \in F$ and $k \notin F$.

Proposition 9. Let $C$ and $C^{\prime}$ be two consecutive minimal covers with respect to constraint (1) such that $E_{M}(C) \neq \emptyset$ and $E_{M}\left(C^{\prime}\right) \neq \emptyset$. Then $E_{M}(C) \cap E_{M}\left(C^{\prime}\right) \neq \emptyset$ if and only if $C=C^{\prime}$.

Proof. $(\Rightarrow)$ Let $F \in E_{M}(C) \cap E_{M}\left(C^{\prime}\right)$. If $F$ is a consecutive cover, by Lemma 6 we have that $E(C)=E\left(C^{\prime}\right)$ and, so, $C=C^{\prime}$. If $F$ is a non-consecutive cover, by Lemma 6 and Proposition 8 there exist $l \in\{\underline{\alpha}(C), \ldots, \bar{\alpha}(C)\}, k \in m(C), l^{\prime} \in\left\{\underline{\alpha}\left(C^{\prime}\right), \ldots, \bar{\alpha}\left(C^{\prime}\right)\right\}$ and $k^{\prime} \in m\left(C^{\prime}\right)$ such that $(E(C) \backslash\{k\}) \cup\{l\}=\left(E\left(C^{\prime}\right) \backslash\left\{k^{\prime}\right\}\right) \cup\left\{l^{\prime}\right\}$, hence $C=C^{\prime}$.

$(\Leftarrow)$ Trivial.

Algorithm 2 identifies all consecutive minimal covers with respect to constraint (1) and, for each of them, determines the covers in $E_{M}(C)$ by using Proposition 8. (Note that, by Proposition 9, all of the maximal covers obtained by Algorithm 2 will be distinct.)

\section{Algorithm 2.}

Step 1. Set $h=0$ and $\bar{\gamma}(C)=\min \left\{l \in J_{0} \mid \sum_{j=1}^{l} a_{j}>b\right\}$. 
Step 2. Set $\underline{\gamma}(C)=\max \left\{l \in J_{0} \mid \sum_{j=l}^{\bar{\gamma}(C)} a_{j}>b\right\}$ and $C=\{\underline{\gamma}(C), \ldots, \bar{\gamma}(C)\} \quad(C$ is a consecutive minimal cover with respect to constraint (1)).

Step 3. Set $h=h+1$ and $C_{h}=E(C)$. If $\underline{\gamma}(C)=1$, set $\underline{\alpha}(C)=1$ and go to Step 8 . Otherwise, set $\bar{\alpha}(C)=\underline{\gamma}(C)-1$.

Step 4. Set $\underline{\alpha}(C)=\min \left\{l \in J_{0} \mid l \leq \underline{\gamma}(C), a_{l}+\sum_{j \in C \backslash\{\underline{\gamma}(C)\}} a_{j}>b\right\}$. If $\bar{\alpha}(C)<\underline{\alpha}(C)$, go to Step 8. Otherwise, set $\bar{\gamma}(m(C))=\max \left\{j \in C \backslash\{\bar{\gamma}(C)\} \mid a_{j}=a_{\underline{\gamma}(C)}\right\}$ and $k=\underline{\gamma}(C)$.

Step 5. Set $l=\underline{\alpha}(C)$.

Step 6. Set $h=h+1$ and $C_{h}=(E(C) \backslash\{k\}) \cup\{l\}$. If $l<\bar{\alpha}(C)$, set $l=l+1$ and repeat Step 6.

Step 7. If $k<\bar{\gamma}(m(C))$, set $k=k+1$ and go to Step 5 .

Step 8. If $\bar{\gamma}(C)=n_{0}$, STOP (all consecutive minimal covers with respect to constraint (1) have been identified).

Step 9. Set $\underline{\gamma}(C)=\underline{\gamma}(C)+1$ and $\bar{\gamma}(C)=\bar{\gamma}(C)+1$. If $\{\underline{\gamma}(C), \ldots, \bar{\gamma}(C)\}$ is a minimal cover with respect to constraint $(1)$, set $\bar{\alpha}(C)=\underline{\alpha}(\bar{C})-1$ and $C=\{\underline{\gamma}(C), \ldots, \bar{\gamma}(C)\}$; go to Step 4. Otherwise, go to Step 2.

ExAmPle 2. Consider the 0-1 knapsack constraint

$$
2 x_{1}+3 x_{2}+4 x_{3}+6 x_{4}+8 x_{5} \leq 12
$$

Algorithm 2 proceeds as follows:

Step 1. $h=0, \bar{\gamma}(C)=4$

Step 2. $\gamma(C)=2, C=\{2,3,4\}$

Step 3. $\bar{h}=1, C_{1}=\{2,3,4,5\}, \bar{\alpha}(C)=1$

Step 4. $\underline{\alpha}(C)=2$

Step 9. $\gamma(C)=3, \bar{\gamma}(C)=5$

Step 2. $\bar{\gamma}(C)=4, C=\{4,5\}$

Step 3. $\bar{h}=2, C_{2}=\{4,5\}, \bar{\alpha}(C)=3$

Step 4. $\underline{\alpha}(C)=4$

Accordingly, the maximal covers from the set of covers implied by constraint (3) that have been identified by Algorithm 2 are $C_{1}=\{2,3,4,5\}$ and $C_{2}=\{4,5\}$, and their induced inequalities are:

$$
\begin{array}{r}
x_{2}+x_{3}+x_{4}+x_{5} \leq 2 \\
x_{4}+x_{5} \leq 1
\end{array}
$$

(Note that Algorithm 2 only obtains two of the four maximal covers from the set of covers implied by constraint (3), see Example 1.) 


\section{Computational experiments}

In this section a computational comparison of Algorithms 1 and 2 is reported. The implementation platform is Microsoft FORTRAN PowerStation Optimizing Compiler v4.0 and Pentium III, $1000 \mathrm{Mhz}, 256 \mathrm{Mb}$ RAM.

The coefficients $\left\{a_{j}\right\}_{j \in J_{0}}$ have been randomly generated so that $a_{j} \in\{1, \ldots, 1000\}$ $\forall j \in J_{0}$. The Quicksort method has been used for sorting $\left\{a_{j}\right\}_{j \in J_{0}}$ in non-decreasing order, see subroutines "sort" and "indexx" in Sections 8.2 and 8.4 of [10] respectively. Several right-hand-sides

$b \in\left\{a_{n_{0}}, \ldots, \sum_{j \in J_{0}} a_{j}-1\right\}$ have been considered. For each of them, we define $\rho=\frac{b-a_{n_{0}}}{\sum_{j \in J_{0} \backslash\left\{n_{0}\right\}} a_{j}-1}$. (Note that $\rho \in[0,1]$ if $\sum_{j \in J_{0} \backslash\left\{n_{0}\right\}} a_{j} \neq 1$.)

The tables below show the values of $n_{0}, b$ and $\rho$, the number of maximal covers identified by Algorithms 1 and 2, and the CPU time expressed in seconds. The column headed "\%" gives the percentage of maximal covers identified by Algorithm 2.

We can observe in Table 1 that the computational effort is practically null for both algorithms. Moreover, since the number of maximal covers from the set of covers implied by the constraints $\sum_{j \in J_{0}} a_{j} x_{j} \leq b$ is not large, it is convenient to identify all of them. So, it is advisable to apply Algorithm 1 independently of the value of $b$.

In Tables 2 and 3 it is worthy of note the large number of maximal covers that can be obtained from a constraint with relatively few variables, and the low percentage of them that Algorithm 2 identifies.

Table 1

\begin{tabular}{||c||c||c|c|c||c|c||}
\hline \multicolumn{2}{||c||}{$n_{0}=10$} & \multicolumn{2}{c||}{ Number of maximal covers } & \multicolumn{2}{c||}{ CPU time } \\
\hline$b$ & $\rho$ & Alg. 1 & Alg. 2 & $\%$ & Alg. 1 & Alg. 2 \\
\hline 775 & 0.0000 & 16 & 10 & 62.5000 & 0.00 & 0.00 \\
1100 & 0.1031 & 19 & 9 & 47.3684 & 0.00 & 0.00 \\
1420 & 0.2047 & 32 & 9 & 28.1250 & 0.00 & 0.00 \\
1750 & 0.3094 & 31 & 8 & 25.8065 & 0.00 & 0.00 \\
2050 & 0.4046 & 28 & 9 & 32.1429 & 0.00 & 0.00 \\
2360 & 0.5030 & 33 & 3 & 9.0909 & 0.00 & 0.00 \\
2670 & 0.6014 & 15 & 3 & 20.0000 & 0.00 & 0.00 \\
3000 & 0.7061 & 20 & 3 & 15.0000 & 0.00 & 0.00 \\
3300 & 0.8013 & 6 & 4 & 66.6667 & 0.00 & 0.00 \\
3770 & 0.9505 & 3 & 2 & 66.6667 & 0.00 & 0.00 \\
\hline
\end{tabular}

Table 2 


\begin{tabular}{||c||c||r|c|c||c|c||}
\hline \multicolumn{2}{||c||}{$n_{0}=25$} & \multicolumn{2}{c||}{ Number of maximal covers } & \multicolumn{2}{c||}{ CPU time } \\
\hline$b$ & $\rho$ & Alg. 1 & Alg. 2 & $\%$ & Alg. 1 & Alg. 2 \\
\hline 991 & 0.0000 & 204 & 24 & 11.7647 & 0.00 & 0.00 \\
2200 & 0.1032 & 4772 & 38 & 0.7963 & 0.00 & 0.00 \\
3400 & 0.2057 & 42175 & 45 & 0.1067 & 0.00 & 0.00 \\
4600 & 0.3081 & 151615 & 39 & 0.0257 & 0.05 & 0.00 \\
5700 & 0.4021 & 281657 & 32 & 0.0114 & 0.17 & 0.00 \\
6900 & 0.5045 & 317317 & 35 & 0.0110 & 0.22 & 0.00 \\
8100 & 0.6070 & 189657 & 28 & 0.0148 & 0.16 & 0.00 \\
9200 & 0.7009 & 67405 & 20 & 0.0297 & 0.06 & 0.00 \\
10400 & 0.8034 & 10324 & 11 & 0.1065 & 0.00 & 0.00 \\
12200 & 0.9571 & 45 & 2 & 4.4444 & 0.00 & 0.00 \\
\hline
\end{tabular}

Table 3

\begin{tabular}{|c|c|c|c|c|c|c|}
\hline \multicolumn{2}{|c|}{$n_{0}=40$} & \multicolumn{3}{|c|}{ Number of maximal covers } & \multicolumn{2}{|c|}{ CPU time } \\
\hline $\bar{b}$ & $\rho$ & Alg. 1 & Alg. 2 & $\%$ & Alg. 1 & Alg. 2 \\
\hline 970 & 0.0000 & 309 & 41 & 13.2686 & 0.00 & 0.00 \\
\hline 3000 & 0.1092 & 888720 & 103 & 0.0116 & 0.71 & 0.00 \\
\hline 4800 & 0.2060 & 56852218 & 136 & 0.0002 & 25.16 & 0.00 \\
\hline 6600 & 0.3028 & 846894137 & 140 & 0.0000 & 346.25 & 0.00 \\
\hline 8500 & 0.4050 & 4020565514 & 121 & 0.0000 & 1596.52 & 0.00 \\
\hline 10300 & 0.5019 & 5817165247 & 96 & 0.0000 & 2317.64 & 0.00 \\
\hline 12200 & 0.6041 & 2710517480 & 103 & 0.0000 & 1085.06 & 0.00 \\
\hline 14000 & 0.7009 & 399908945 & 60 & 0.0000 & 175.16 & 0.00 \\
\hline 16000 & 0.8085 & 8954344 & 22 & 0.0002 & 5.82 & 0.00 \\
\hline 18800 & 0.9591 & 240 & 5 & 2.0833 & 0.00 & 0.00 \\
\hline
\end{tabular}

For some of the values of $b$ that have been considered in Tables 2 and 3, the number of maximal covers from the set of covers implied by the constraint $\sum_{j \in J_{0}} a_{j} x_{j} \leq b$ is small enough to keep them stored in the computer's memory. In these cases it is preferable to apply Algorithm 1, since it requires little computational effort and guarantees the identification of all of the maximal covers.

For the rest of the values of $b$, the number of covers that Algorithm 2 identifies is, in general, small. Consequently, it would be advisable to limit the number of covers to be obtained by Algorithm 1 so that these covers could be stored in the computer's memory.

\section{Conclusions}

In this paper two procedures for identifying maximal cover from the set of covers implied by a $0-1$ knapsack constraint have been presented. The first one identifies all of them. The second one is an improvement on a procedure developed by Dietrich, Escudero, Garín and Pérez in 1993 that only identifies certain maximal covers. We have shown that the 
maximal covers obtained by the second procedure can be a very small fraction of the whole set of maximal covers. Thus, we propose to limit the number of maximal covers that the first procedure is allowed to identify. We believe that embedding this procedure in a branch-and-cut framework for knapsack constraint tightening and direct cut appending can pay the effort.

\section{References}

[1] Dietrich, B.L.; Escudero, L.F.; Garín, A.; Pérez, G. (1993) "O(n) procedures for identifying maximal cliques and non-dominated extensions of consecutive minimal covers and alternates", Top 1(1): 139-160.

[2] Escudero, L.F.; Martello, S.; Toth, P. (1998) "On tightening 0-1 programs based on extensions of pure 0-1 knapsack and subset-sum problems", Annals of Operations Research 81: 379-404.

[3] Escudero, L.F.; Muñoz, S. (1998) "On characterizing tighter formulations for 0-1 programs", European Journal of Operational Research 106(1): 172-176.

[4] Escudero, L.F.; Muñoz, S. (2002) "On characterizing maximal covers", Investigación Operacional (to appear).

[5] Hoffman, K.L.; Padberg, M. (1991) "Improving LP-representations of zero-one linear programs for branch-and-cut", ORSA Journal on Computing 3(2): 121-134.

[6] Johnson, E.L.; Nemhauser, G.L.; Savelsbergh, M.W.P. (2000) "Progress in linear programming-based algorithms for integer programming: An exposition", INFORMS Journal on Computing 12(1): 2-23.

[7] Muñoz, S. (1995) "A correction of the justification of the Dietrich-Escudero-GarínPérez $O(n)$ procedures for identifying maximal cliques and non-dominated extensions of consecutive minimal covers and alternates", Top 3(1): 161-165.

[8] Muñoz, S. (1999) Reforzamiento de Modelos en Programación Lineal 0-1. Tesis Doctoral, Universidad Complutense de Madrid, Madrid.

[9] Nemhauser, G.L.; Wolsey, L.A. (1998) Integer and Combinatorial Optimization. John Wiley, New York.

[10] Press, W.H.; Teukolsky, S.A.; Vetterling, W.T.; Flannery, B.P. (1992) Numerical Recipes in FORTRAN. The Art of Scientific Computing. Cambridge University Press.

[11] Savelsbergh, M.W.P. (1994) "Preprocessing and probing techniques for mixed integer programming problems", ORSA Journal on Computing 6(4): 445-454. 